\title{
Implementation of Dimming controlled Visible Light Communication using Raspberry $\mathrm{Pi}$
}

\section{Sandip Das ( $\square$ info.sandipec@gmail.com )}

University of Engineering \& Management, Jaipur https://orcid.org/0000-0001-5150-6763

\section{Biswajit Jana}

University of Engineering \& Management, Jaipur

\section{Soumitra Kumar Mandal}

National Institute of Technical Teachers' Training and Research Kolkata

\section{Research Article}

Keywords: Visible Light Communication, MH-HPPM, Dimming, Raspberry Pi

Posted Date: July 19th, 2021

DOl: https://doi.org/10.21203/rs.3.rs-559095/v1

License: (1) This work is licensed under a Creative Commons Attribution 4.0 International License. Read Full License

Version of Record: A version of this preprint was published at Optical and Quantum Electronics on November 19th, 2021. See the published version at https://doi.org/10.1007/s11082-021-03362-4. 


\title{
Implementation of Dimming controlled Visible Light Communication using Raspberry Pi
}

\author{
Sandip Das $^{1 * \dagger}$, Biswajit Jana ${ }^{2^{* *}}$ and Soumitra Kumar Mandal ${ }^{3 \ddagger}$ \\ ${ }^{1,2}$ Electronics and Communication, University of Engineering \& Management, Jaipur, \\ Rajasthan, India \\ ${ }^{3}$ Electrical Engineering, National Institute of Technical Teachers' Training \& Research, \\ Kolkata, West Bengal, India
}

\begin{abstract}
Visible light communication (VLC) is a technology of wireless optical communication, which combines lighting and communication simultaneously. In indoor VLC system, both data communication and lighting quality plays an important role and must be considered. Thus, to achieve the desired levels of illumination, dimming control in VLC is an efficient technology. In this paper, the authors have designed and implemented a dimming control VLC system employing multi header-hybrid pulse position modulation (MH-HPPM) to achieve dimming functionality. MH-HPPM supports various dimming levels and also doesn't have flicker problems. In this paper, the authors present the implementation of MHHPPM based VLC system using Raspberry pi. The prototype is designed using low-cost commodity hardware. Comprehensive experiments are carried out to evaluate the performance of MH-HPPM based VLC under dimming levels 0.25, 0.5 and 0.75 . The results demonstrate that designed prototype supports a communication distance up to $3 \mathrm{~m}$ for dimming level 0.25 and $3.6 \mathrm{~m}$ for dimming level 0.5 and 0.75 . It is also observed that MH-HPPM based VLC system also achieves and maintains better throughput for different incidence angle.
\end{abstract}

Key words: Visible Light Communication, MH-HPPM, Dimming, Raspberry Pi

\section{Introduction}

Visible light communication (VLC) is the subset of optical wireless communication technology which uses visible light as the source to transmit high speed data. This data is transmitted by modulating the intensity of visible light source and is received by the photodiode in the receiver side. The data received is then readily transformed into the forms readable by the end users. VLC technology becomes a viable option with its vast visible light spectrum, which carries $300 \mathrm{THz}$ of license free bandwidth compared to the dwindling RF spectrum. The characteristics which make VLC technology unique in itself are signal confinement, non-line of sight and safe in hazardous environments (hazardous environments means hospitals, air planes, mines etc., where RF signal may be perceived as hazard).

The visible light source, i.e., LED, transmits data continuously by turning LED's ON or OFF depending upon the digital data sent into it. Thus, the distribution of digital data, 1's and 0's in a transmitted data frame affects the illumination and stabilization of the light. Hence, the VLC physical layer faces two main obstacles namely flickering and dimming control [1]-[3]. As identified by IEEE 802.15.7 task group dimming control is one of the key challenges in VLC systems. Though dimming functionality provides energy saving but it harms the transmission rate. Hence, to communicate with an optimum transmission rate through a VLC link, different dimming methods are proposed in [4]-[10] based on coding and modulation techniques.

Different modulation schemes like on-off keying (OOK), pulse position modulation (PPM) and its variants like variableOOK (VOOK), variable-PPM (VPPM) and multi pulse position modulation (MPPM) supports dimming functionality. Other variants of PPM which supports dimming are overlap-PPM (OPPM), inverse-PPM (I-PPM) and multi headerhybrid PPM (MH-HPPM) is reported in [11]-[13]. In [13], MH-HPPM is proposed and its performance is compared with PPM and PPM variant modulation schemes. It is reported in [13] that MH-HPPM utilizes the property of PPM and Inverse-PPM (IPPM) to achieve a varied range of dimming values and has better performance compared to OOK, PPM, digital pulse interval modulation (DPIM), dual header-PIM (DH-PIM) and multi header-PIM (MH-PIM).

There has been literatures which has reported the use of Raspberry pi for designing VLC system [14]-[18]. In [14] a prototype is proposed which is used to transmit images using OOK based modulation scheme, in [15] Raspberry pi is

*Corresponding author.

†E-mail: info.sandipec@gmail.com

ORCID ID: 0000-0001-5150-6763 
used to design a prototype of position detection system which also uses OOK based modulation scheme. In [16] a CSKCDMA based VLC is reported. In this paper, the authors have designed a prototype to implement and test the dimming functionality of the MH-HPPM proposed in [13] for transmitting data using Raspberry Pi. The prototype is designed such that it can transmit input text of any length from transmitter device to receiver device using VLC technology by maintaining the dimming value defined by the user.

\section{System Overview}

The system architecture overview of the VLC system using Raspberry pi is shown in fig.1. A typical VLC system comprises of transmitter and receiver. The transmitter of the VLC system works as:

1. Upon receiving the text data, the user is prompted to set the dimming target value from the available set of dimming values.

2. In the MH-HPPM encoder, the ASCII value of each letter, spaces, alphanumeric values or special characters are converted into binary vector which forms the message.

2.1. Depending on the target dimming value the binary message vector is divided into blocks, where the block size varies according to dimming value and then each block is encoded into MH-HPPM frame.

2.2. The MH-HPPM frame generated using software (from PC) consists of array of 1's and 0's and are sent to Raspberry pi.

3. The array of bits is sent through the GPIO pins of Raspberry pi to the LED driver circuit which drives (modulates) the

LED. If bit is ' 1 ' LED turns ON, if bit is ' 0 ' the LED is turned off at a very fast switching rate.

At the receiver, the photodiode detects the light signal. The MH-HPPM decoder in the receiver section decodes each frame of MH-HPPM into array of binary vector which is then converted into corresponding text data.

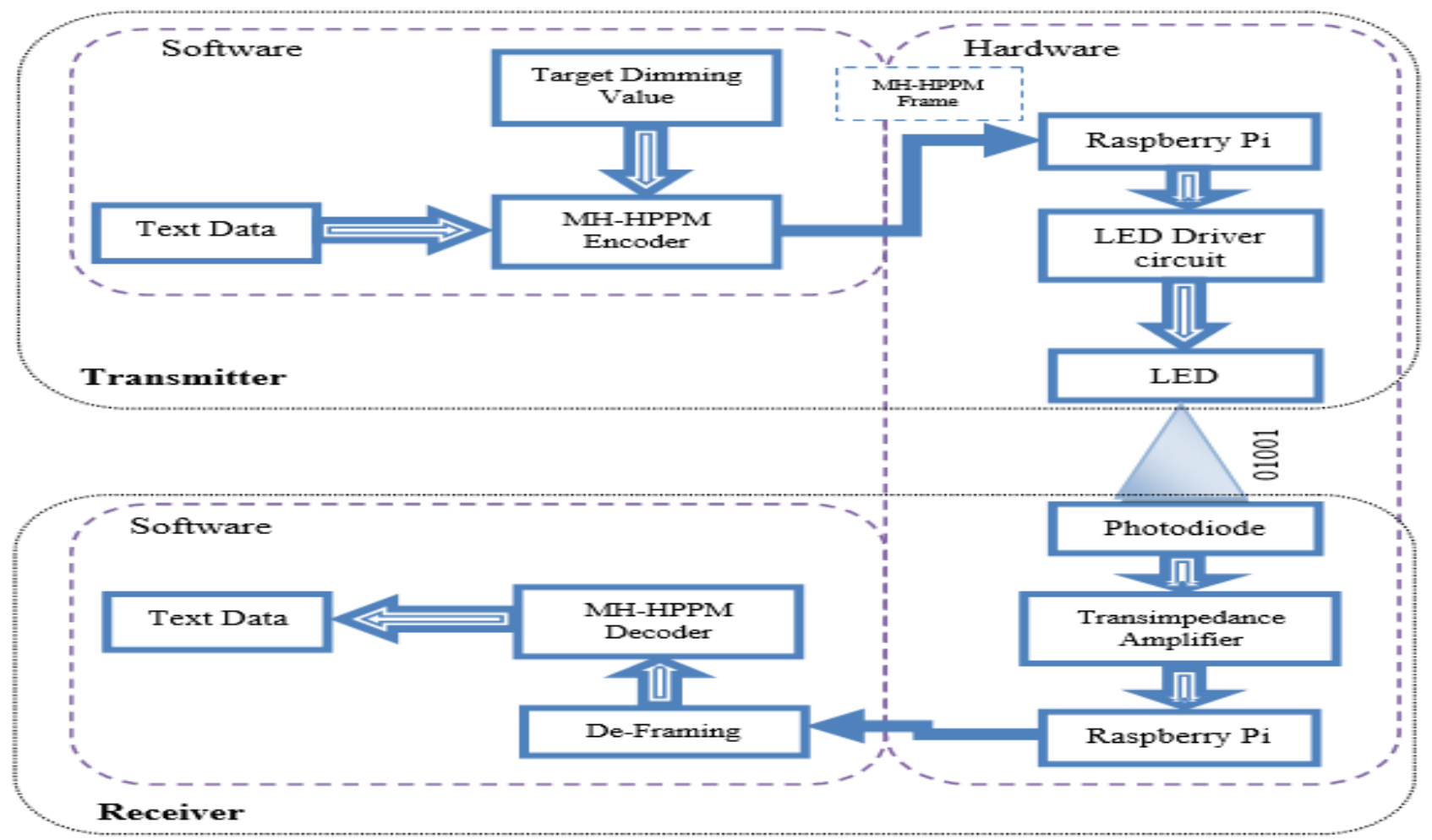

Fig. 1 System architecture of dimming controlled MH-HPPM VLC system

\section{MH-HPPM system design}

MH-HPPM is capable of providing dimming functionality and the range of dimming values depends on the block size []. Before the MH-HPPM frame generation and dimming functionality is discussed, the key definitions that are used throughout the work is given as follows:

- Block size- denoted as $K$. Any random data sequence is split into block of $K$ bits depending on the dimming value.

- Time slot- denoted as $t_{\text {slot }}$. For any specific hardware, $t_{\text {slot }}$ is fixed. Hence, system can turn LED on or off at a 
maximum rate of $1 / t_{\text {slot }}$.

- Symbol- a group of $N$ time slots consisting of either on or off states which together represents one or several data bits.

- $\quad$ Symbol duration- denoted as $T$, where $T=N . t_{\text {slot }}$. The value of $N$ depends on the dimming target value and block size.

- Dimming value- denoted as $\alpha$. It indicates the intensity/brightness level of the LED. Mathematically, it can be represented as:

$$
\alpha=\frac{\text { number of on states }}{N}
$$

The range of $\alpha$ varies from [0,1]. For example, $\alpha=0.5$ means brightness level is $50 \%$.

As proposed in [13], MH-HPPM frame can be generated as follows:

1. Depending on the dimming target value $\alpha$, any random data sequence is split into a block of $K$ bits of information data given as $b=\left[b_{K}, b_{K-1}, \ldots, b_{3}, b_{2}, b_{1}\right]$. The dimming target value $\alpha$, and the equivalent block length, $K$, of information data are listed in Table I.

2. MH-HPPM frame consists of two parts- the header part, $d_{h}$ and the message part, $m$. Depending on the dimming target value $\alpha$, the message part, $m$, is generated from $\left[b_{2}, b_{1}\right]$ and its decimal equivalent is mapped either into PPM or inverse PPM. Thus, the length of the message part of the MH-HPPM frame is always $m_{l}=4$.

3. Similarly, depending on the dimming target value $\alpha$, the header part, $d_{h}$, is generated from $\left[b_{K}, b_{K-1}, \ldots, b_{3}\right]$ and its decimal equivalent is mapped either as PPM or inverse PPM. Thus, the length of the dimming header of the MH-HPPM frame is $d_{l}=2^{K-2}$.

Hence, the total time slots of MH-HPPM frame are $N=d_{l}+m_{l}=2^{K-2}+4$. Therefore, the dimming value can now be represented as:

$$
\alpha=\frac{\text { number of oN states }}{2^{K-2}+4}
$$

TABLE I

DIMMING TARGET VALUE, AND CORRESPONDING BLOCK LENGTH $K$

\begin{tabular}{|c|c|c|c|c|c|c|c|c|c|c|c|c|c|}
\hline Dimming target value, $\alpha$ & 0.33 & 0.67 & 0.25 & 0.5 & 0.75 & 0.17 & 0.33 & 0.67 & 0.83 & 0.1 & 0.2 & 0.8 & 0.9 \\
\hline Data block length, $K$ & 3 & \multicolumn{3}{|c|}{4} & \multicolumn{3}{|c|}{5} & \\
\hline MH-HPPM frame length & \multicolumn{2}{|c|}{6} & \multicolumn{3}{|c|}{8} & \multicolumn{3}{|c|}{12} & & \\
\hline
\end{tabular}

In fig. 2, the MH-HPPM frame for achieving dimming level of $\alpha=0.25,0.5$ and 0.75 is illustrated for a data of block size $K=4$ and data as ' 1000 '.

Symbol duration, $T$ : Has $N$ slots

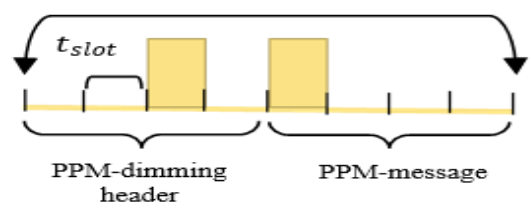

(a)

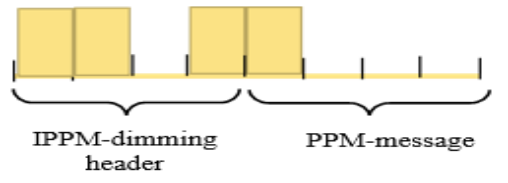

(b)

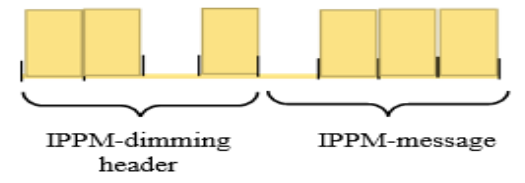

(c)

Fig. 2 Illustration of MH-HPPM frame for achieving dimming level of (a) 0.25 (b) 0.5 (c) 0.75

\subsection{Data Transmission and Frame format}

To enable communication between the transmitter and receiver a frame format must be properly designed. As shown in [13], slots can be extracted/recovered from the received MH-HPPM frame and since multiple headers are used so preamble or synchronization bits isn't needed between consecutive MH-HPPM frames for distinguishing. Hence, the frame format design is optimized as shown in fig. 3 for transmitting data and establishing communication.

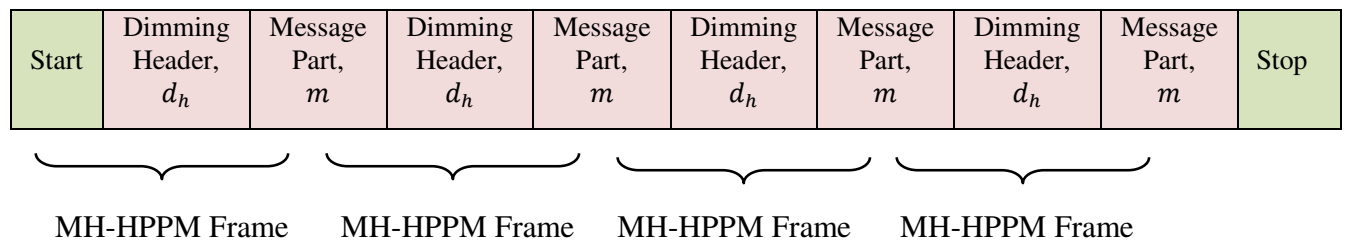

Fig. 3 Frame format to transmit data using MH-HPPM 
Start bits of appropriate size is sent depending on the dimming value to initiate the process of data transmission using MHHPPM frame. The start bits also contain information about the dimming value. Following the start bit is the MH-HPPM data sequence frames. After all the MH-HPPM data sequence, the frame format is terminated by an appropriate size of stop bits to indicate the end of data transmission. After receiving the complete frame, the receiver first extracts the information of dimming value from the start bits which is used to decode the received MH-HPPM frame using appropriate decoding algorithm for the corresponding dimming value.

\section{Implementation}

This section presents the implementation of the dimming-controlled MH-HPPM VLC using raspberry pi which includes both software and hardware. Many a times, it is not convenient to work directly on the Raspberry Pi. Hence, Virtual Network Computing (VNC), a graphical desktop sharing system that allows to remotely control the desktop interface of one computer (running VNC Server) from another computer or mobile device (running VNC Viewer) is used. In this implementation, one PC has been used as a central console to transmit and receive data using VLC and VNC viewer for transmitter is used to control dimming and transmit data at the transmitter and VNC viewer for receiver is used to decode and visualize the received data at the receiver. To connect to the raspberry Pi using VNC Viewer from PC, it is necessary to know the IP address of the Pi's. In the designed prototype, the transmitter Pi's IP is: 169.254.181.83 and, the receiver Pi's IP is: 169.254.126.144, it may vary from PC to PC as the IP's are assigned by the system.

\subsection{Transmitter}

The schematic diagram of the transmitter circuit which contains a Raspberry Pi, a LED driver circuit (using, NPN transistor), a power supply of $12 \mathrm{v}, 2.2 \mathrm{k}$ resistor, COB LED $(12 \mathrm{~V}, 2.4 \mathrm{~W})$ and wires for connection.

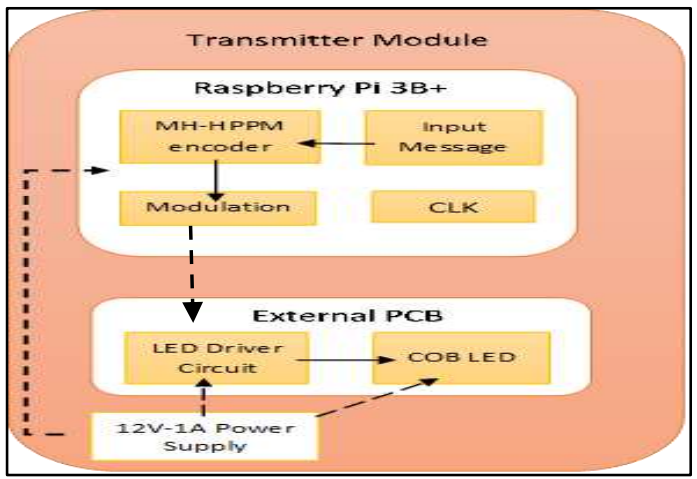

(a)

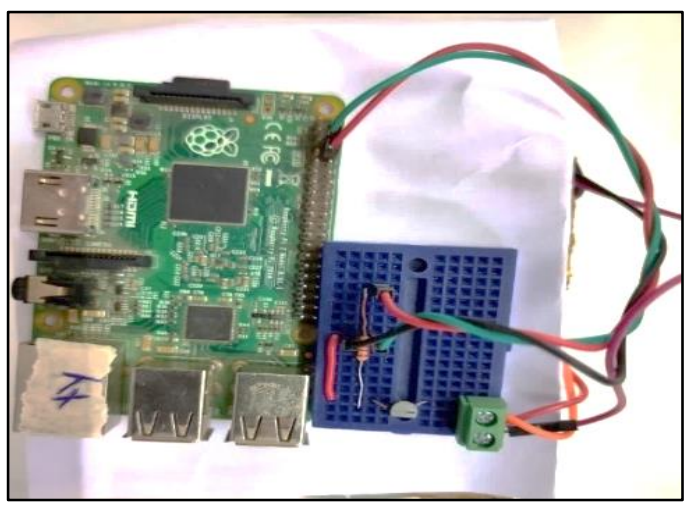

(c)

Fig. 4 (a) Block diagram of transmitter prototype (b) Schematic Diagram of the transmitter module, (c) Top View of the transmitter module, (d) Front View of the transmitter module

The user PC (central console) takes the input data from the user. The prototype is designed to transmit only alphanumeric data using dimming controlled MH-HPPM. The MH-HPPM encoding is performed using python programming. The user in the transmitter side can opt for any dimming value $(0.25,0.5$ or 0.75$)$ and the python code will generate corresponding MH-HPPM dimming frame as shown in algorithm 1. Fig. 5 demonstrates the MH-HPPM frame generated for an input character "A" when dimming value $\alpha=0.25$ using python code.

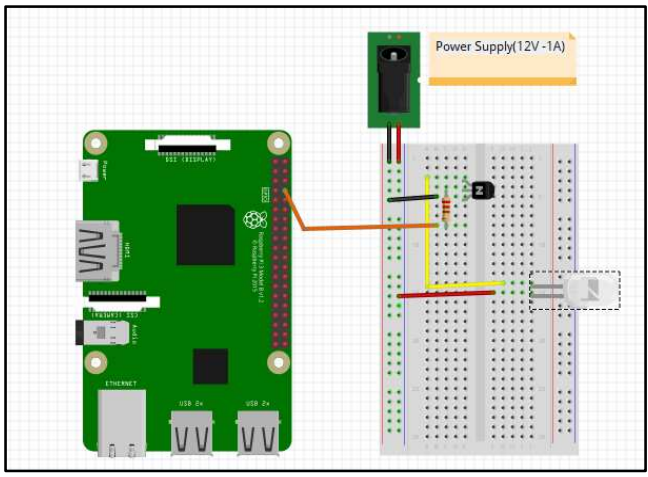

(b)

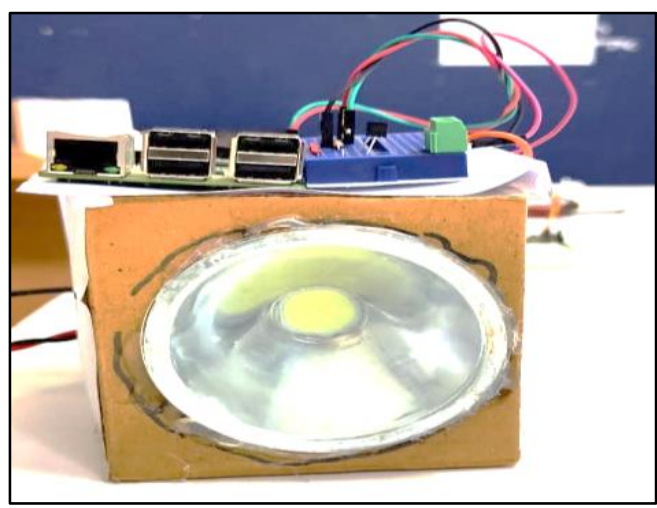

(d) 


\section{Algorithm 1:}

Require:

Step 1: Get input binary data and dimming target value $\alpha$

Step 2: if $\alpha=0.25$, set START $=$ [11111000]

else if $\alpha=0.5$, set START $=[11111001]$

else if $\alpha=0.75$, set START $=[11111010]$

Step 3: Set END= [11111111]

Step 4: if $\alpha=0.25$ then $K=4$ and $b=\left[b_{4}, b_{3}, b_{2}, b_{1}\right]$

$d_{4}=\overline{b_{4}} \cdot \overline{b_{3}}, d_{3}=\overline{b_{4}} \cdot b_{3}, d_{2}=b_{4} \cdot \overline{b_{3}}, d_{1}=b_{4} \cdot b_{3}$

$m_{4}=\overline{b_{2}} \cdot \overline{b_{1}}, m_{3}=\overline{b_{2}} \cdot b_{1}, m_{2}=b_{1} \cdot \overline{b_{2}}, m_{1}=b_{1} \cdot b_{2}$

$d_{h}=\left[d_{4}, d_{3}, d_{2}, d_{1}\right] \quad m=\left[m_{4}, m_{3}, m_{2}, m_{1}\right]$

else if $\alpha=0.5$ then $K=4$ and $b=\left[b_{4}, b_{3}, b_{2}, b_{1}\right]$

$d_{4}=\left(b_{4} \oplus b_{3}\right)+b_{4} \cdot b_{3}, d_{3}=\overline{b_{4}} \cdot \overline{b_{3}}+b_{4}, d_{2}=\overline{b_{4}} \cdot \overline{b_{3}}+b_{3}, d_{1}=\left(b_{4} \oplus b_{3}\right)+\overline{b_{4}} \cdot \overline{b_{3}}$

$m_{4}=\overline{b_{2}} \cdot \overline{b_{1}}, m_{3}=\overline{b_{2}} \cdot b_{1}, m_{2}=b_{1} \cdot \overline{b_{2}}, m_{1}=b_{1} \cdot b_{2}$

$d_{h}=\left[d_{4}, d_{3}, d_{2}, d_{1}\right] \quad m=\left[m_{4}, m_{3}, m_{2}, m_{1}\right]$

else if $\alpha=0.75$ then $K=4$ and $b=\left[b_{4}, b_{3}, b_{2}, b_{1}\right]$

$d_{4}=\left(b_{4} \oplus b_{3}\right)+b_{4} \cdot b_{3}, d_{3}=\overline{b_{4}} \cdot \overline{b_{3}}+b_{4}, d_{2}=\overline{b_{4}} \cdot \overline{b_{3}}+b_{3}, d_{1}=\left(b_{4} \oplus b_{3}\right)+\overline{b_{4}} \cdot \overline{b_{3}}$

$m_{4}=\left(b_{2} \oplus b_{1}\right)+b_{2} \cdot b_{1}, m_{3}=\overline{\left(b_{2} \oplus b_{1}\right)}+b_{2} \cdot \overline{b_{1}}, m_{2}=\left(b_{2} \oplus b_{1}\right)+\overline{b_{2}} \cdot b_{1}, m_{1}=\left(b_{2} \oplus b_{1}\right)+\overline{b_{2}} \cdot \overline{b_{1}}$

$d_{h}=\left[d_{4}, d_{3}, d_{2}, d_{1}\right] \quad m=\left[m_{4}, m_{3}, m_{2}, m_{1}\right]$

Step 5: MH-HPPM frame $=\left[\right.$ START $d_{h} m$ END]

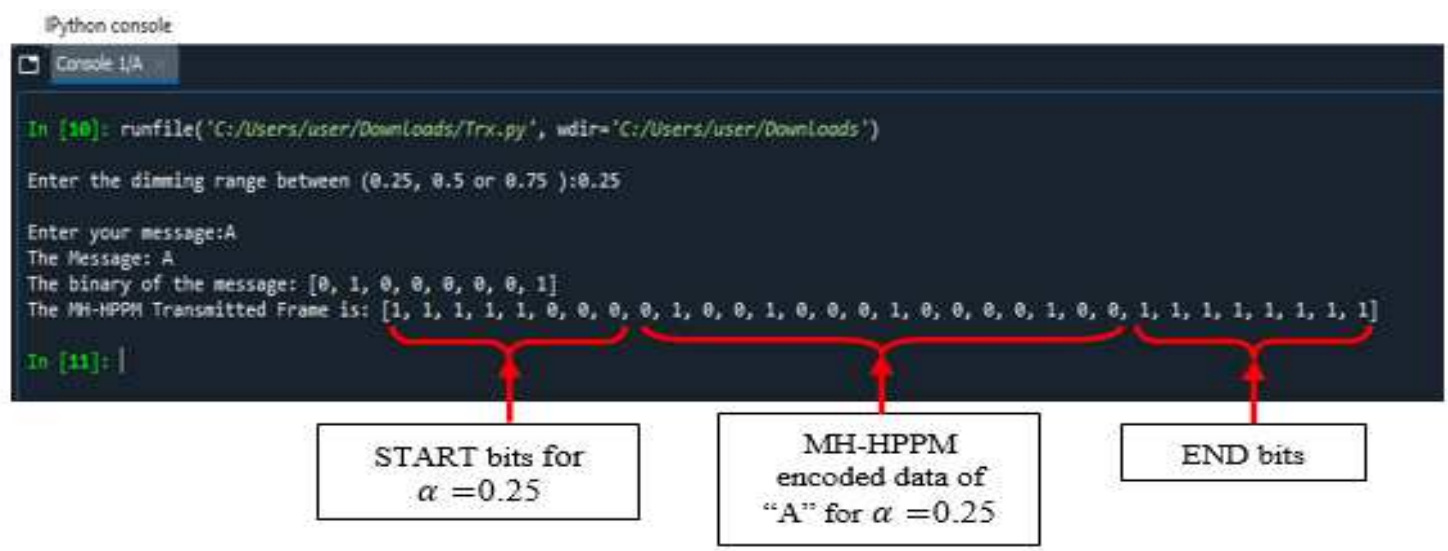

Fig. 5 Showing generation of 0.25 dimmed MH-HPPM frame of input character "A"

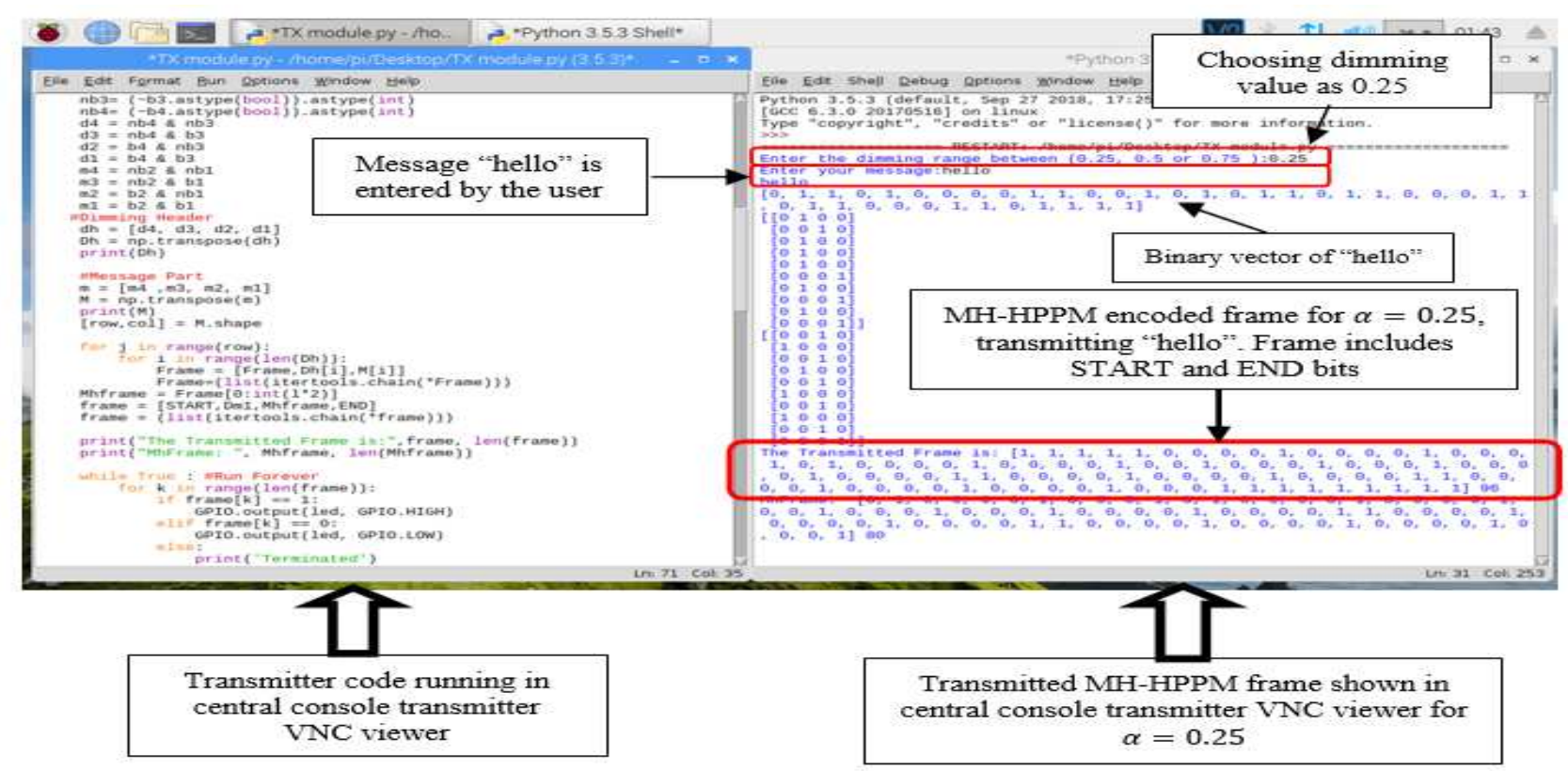

Fig. 6 Showing transmitter code and MH-HPPM transmitted frame $(\alpha=0.25)$ for input data "hello" running in transmitter VNC viewer 
The MH-HPPM encoded frame generated as shown in fig. 6 is sent to LED driver circuit through the RPi GPIO pin and is used drive the LED.

\subsection{Receiver}

The receiver module required more considered selection of components than the transmission module. This is primarily due to the photodiode configuration, the signal amplification and the RPi GPIO requiring protection against over-voltage. The selection of the photodiode drove all of these requirements. Another influence on the choice of the receiver circuit components was to make sure that each stage would not present a limiting factor to the ability of the prototype to receive high speed signals.

The Raspberry Pi for the receiver module is of the same configuration as the transmission module, right down to the cases used to protect the RPi board against damage and contamination. GPIO pin 7 is used to receive the signal from the photodiode circuit. Due to the low power requirements of the amplifier circuit, the on-board $+5 \mathrm{~V}$ GPIO pin 2 is used to power the receiver circuit. The Transimpedance amplifier (TIA) OPA380 is selected partly due to its low drain to reduce the need for an additional power source. The RPi GND pin 6 is used for the circuit to ensure common ground connections. In this prototype, a Hamamatsu S1223 PIN photodiode with a cut-off frequency of $30 \mathrm{MHz}$ and having a high sensitivity in visible to near infrared range is chosen. Also, in order to stop the maximum output $(+4.4 \mathrm{~V})$ from the photodiode and the amplifier reaching the RPi's GPIO's and doing damage, a Zener protection circuit is used to restrict the output of the amplifier to $+3.3 \mathrm{~V}$. The block diagram, schematic and the prototype of the receiver module is shown in fig. 7 .

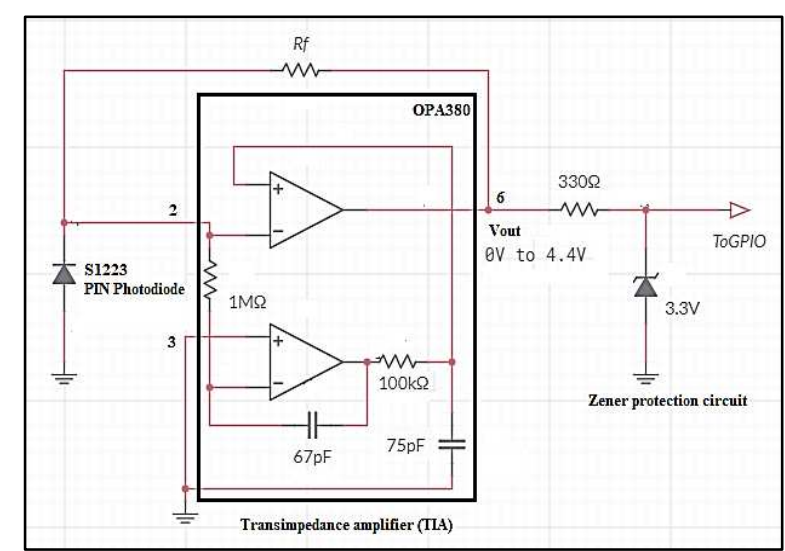

(a)

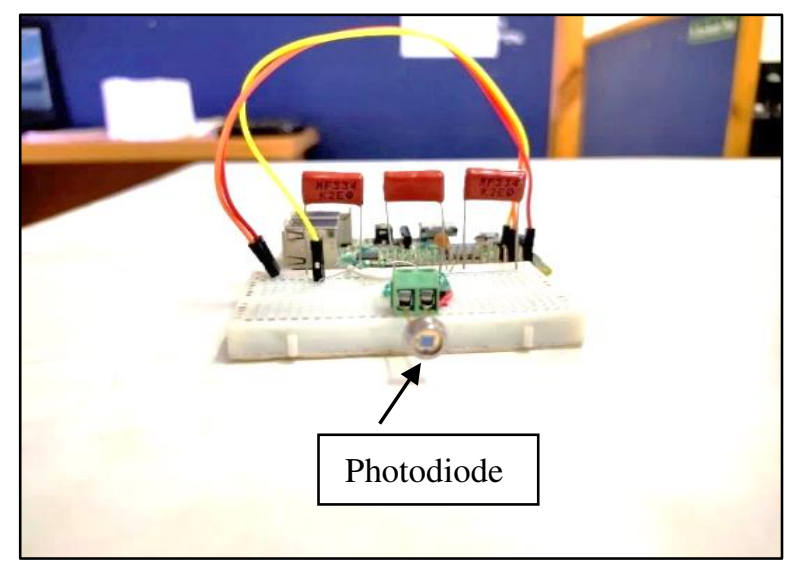

(c)

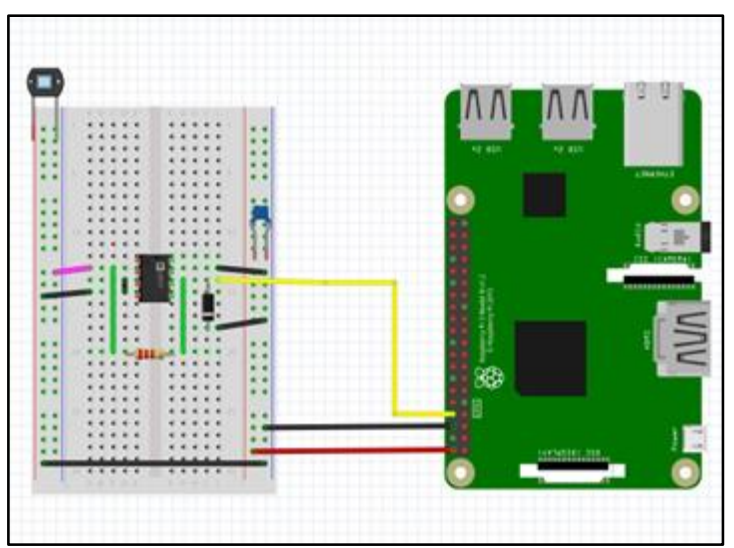

(b)

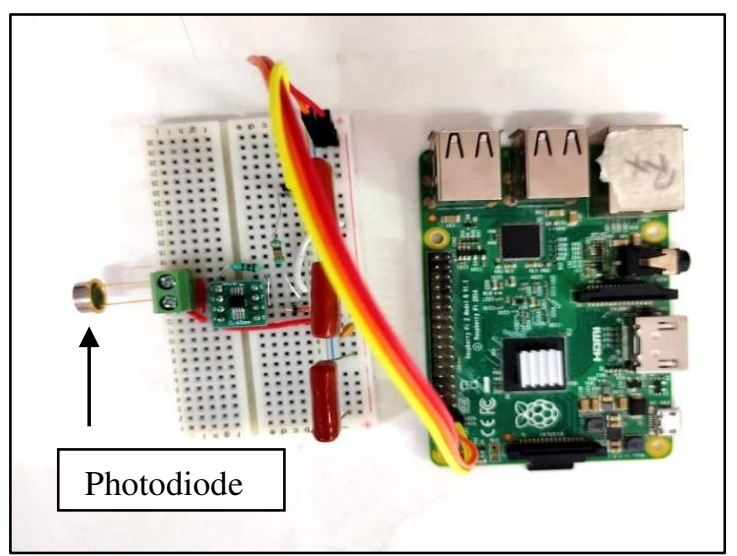

(d)

Fig. 7 (a) Schematic of photodiode, TIA and Zener protection circuit (b) Schematic diagram of the receiver module, (c) Front View of the receiver module, (d) Top View of the receiver module

The photodiode detects the LED's fast ON/OFF switching and sends the corresponding digital bit to the RPi via GPIO pin 7. The received pattern is decoded and converted to text/alphanumeric values. The decoding is carried out using algorithm 2 as shown in fig. 8. In this algorithm it is considered that a MH-HPPM frame (of size 24 bits) for a block of $K=4$ is received. 


\section{Algorithm 2: \\ Require:}

Step 1: received estimated binary signal as $\hat{d}=\left\{\hat{d}_{M}, \ldots \ldots, \hat{d}_{2}, \hat{d}_{1}, \hat{d}_{0}\right\}$ and is appended in an empty list.

Step 2: Set $\alpha=0.25$ if first eight bit $\left\{\hat{d}_{7}, \ldots, \hat{d}_{2}, \hat{d}_{1}, \hat{d}_{0}\right\}$ matches the pattern [11111000]

Else $\alpha$ et $S=0.5$ if first eight bit $\left\{\hat{d}_{7}, \ldots, \hat{d}_{2}, \hat{d}_{1}, \hat{d}_{0}\right\}$ matches the pattern [11111001]

Else $\alpha \mathrm{S}=0.75$ if first eight bit $\left\{\hat{d}_{7}, \ldots, \hat{d}_{2}, \hat{d}_{1}, \hat{d}_{0}\right\}$ matches the pattern [11111010]

Step 3: if $\alpha=0.25$

$$
\begin{aligned}
& \text { then } \hat{b}_{3}=\hat{d}_{15}+\hat{d}_{14}, \hat{b}_{2}=\hat{d}_{15}+\hat{d}_{13}, \hat{b}_{1}=\hat{d}_{9}+\hat{d}_{8} \text { and } \hat{b}_{0}=\hat{d}_{10}+\hat{d}_{8} \\
& \text { else if } \alpha=0.5 \\
& \text { then } \hat{b}_{3}=\hat{d}_{13} \cdot \hat{d}_{13}, \hat{b}_{2}=\hat{d}_{12} \cdot \hat{d}_{14}, \hat{b}_{1}=\hat{d}_{9}+\hat{d}_{8} \text { and } \hat{b}_{0}=\hat{d}_{10}+\hat{d}_{8} \\
& \text { else if } \alpha=0.75 \\
& \text { then } \hat{b}_{3}=\hat{d}_{12} \cdot \hat{d}_{13}, \hat{b}_{2}=\hat{d}_{12} \cdot \hat{d}_{14}, \hat{b}_{1}=\hat{d}_{8} \cdot \hat{d}_{9} \text { and } \hat{b}_{0}=\hat{d}_{8} \cdot \hat{d}_{10} \\
& \hat{b}_{h}=\left\{\hat{b}_{3}, \hat{b}_{2}\right\} \text { and } \hat{b}_{m}=\left\{\hat{b}_{1}, \hat{b}_{0}\right\}
\end{aligned}
$$

Step 4: Terminate if last eight bit $\left\{\hat{d}_{23}, \ldots, \hat{d}_{18}, \hat{d}_{17}, \hat{d}_{16}\right\}$ matched the pattern [11111111]

Output:

Message output as $\hat{b}=\left\{\hat{b}_{h} \hat{b}_{m}\right\}$

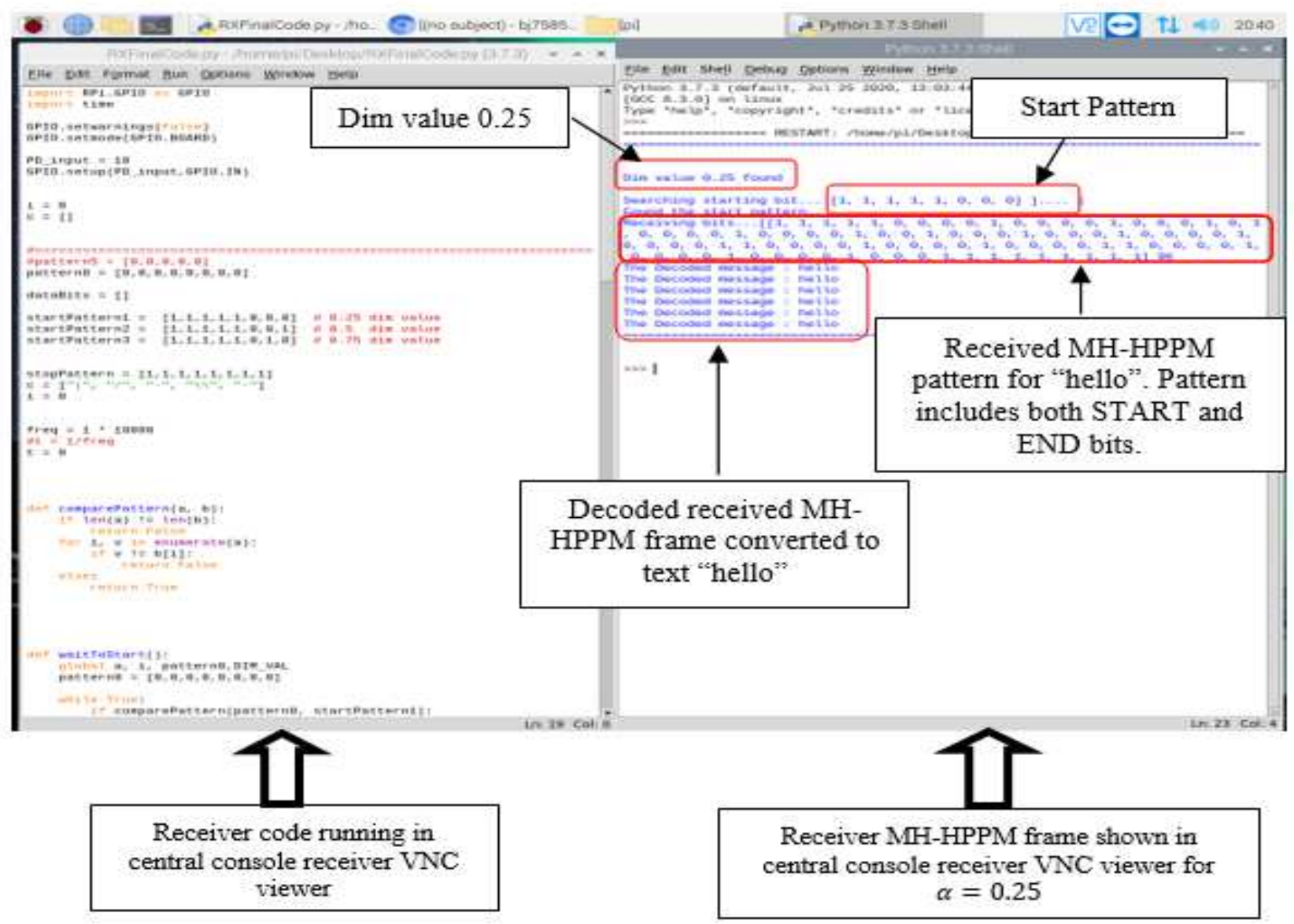

Fig. 8 Showing receiver code, MH-HPPM received frame $(\alpha=0.25)$ and decoded text "hello" running in receiver VNC viewer

\section{Experiment and observations}

Fig. 9 shows the experimental set up of the VLC system prototype. As it is seen from the figure, the central console (PC) is in the middle of the set up running the transmitter VNC viewer and the receiver VNC viewer. 


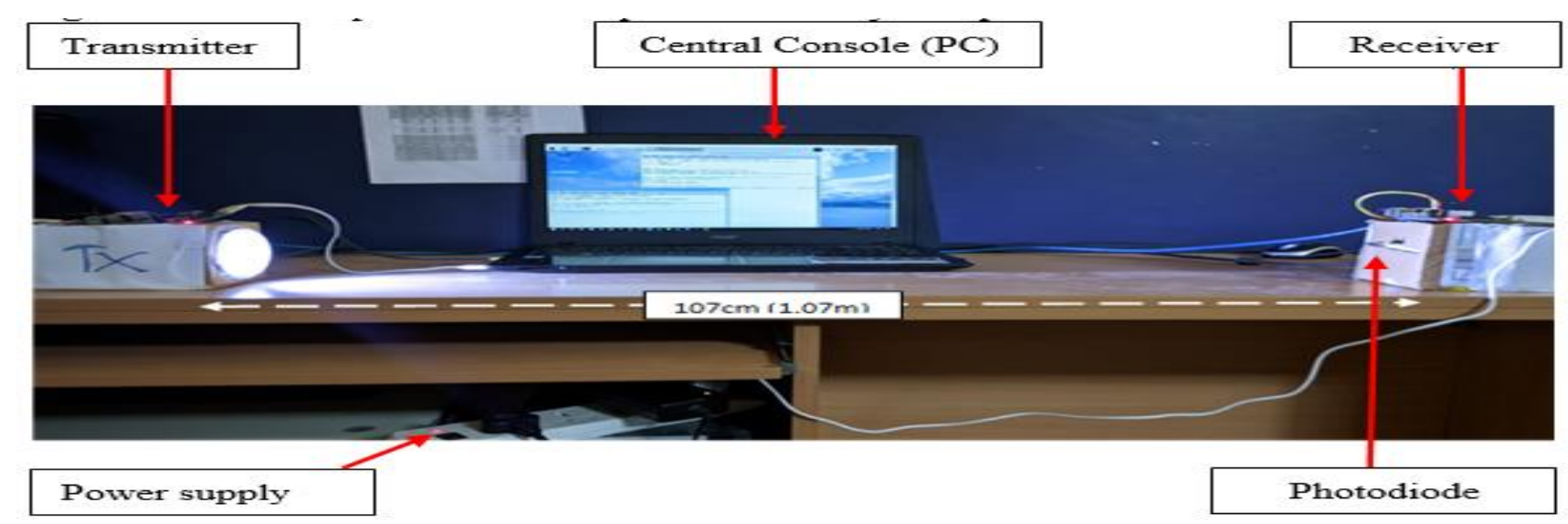

Fig. 9 Experimental set up of VLC system prototype with a dimming value 0.25

As shown in fig. 6, the transmitter VNC viewer executes the transmitter code, allowing the user to select any dimming value between $0.25,0.5$, and 0.75 . Following the selection of the dimming value, the code prompts the user for the data to be transmitted. The data is converted into an MH-HPPM frame and sent to the transmitter LED via RPi. The MH-HPPM frame dims the intensity of the LED to the desired dimming level based on the dimming value selected by the user. During the experiment, it was discovered that the intensity of light with dimming value 0.25 has the lowest intensity and dimming value 0.75 has the highest intensity (as expected). Because the intensity level could not be distinguished from the captured images for different dimming values, the authors measured the light intensity using an Android app called "Lux Light Meter Free," which is available in the Google Play store. The app detects the intensity of light using the smartphone's ambient light sensor. Figure 10 depicts the measurement of light intensity with a smartphone and the "Lux Light Meter Free" app.

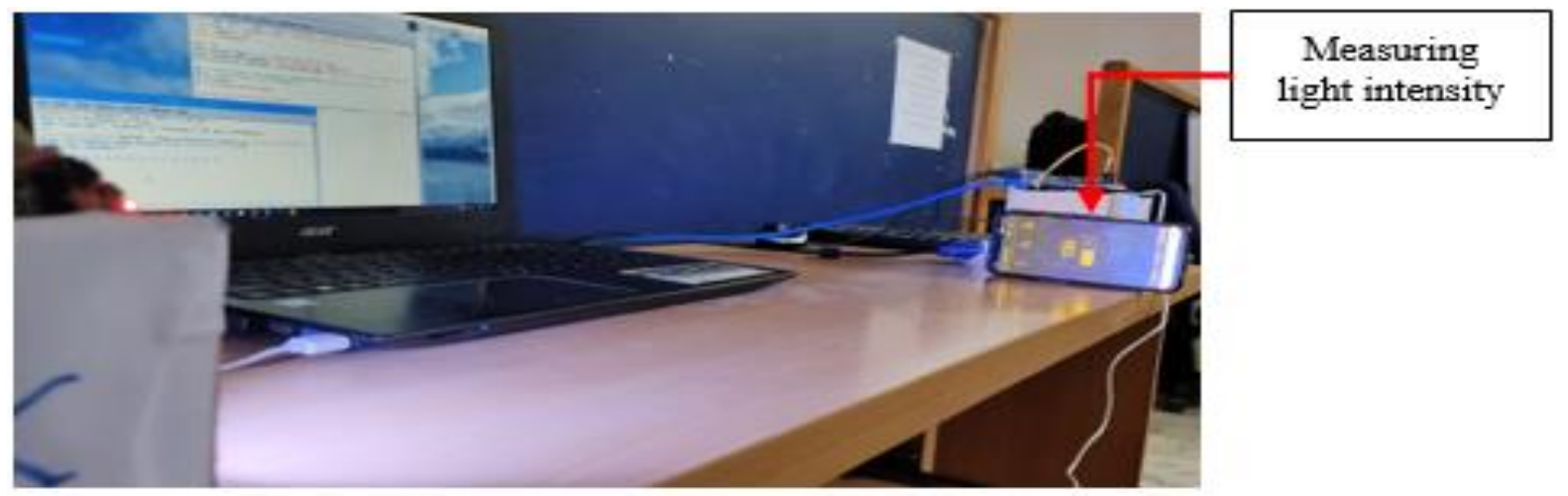

Fig. 10 Measuring light intensity using smartphone and “Lux Light Meter Free” app.

Thus, in this way the ambient light condition of the room and light intensity for different dimming levels have been measured and is shown in fig. 11. Table II lists the measured light intensity for various dimming values. The transmitted and received data for dimming value 0.5 and 0.75 is shown fig. 12 and 13 respectively.

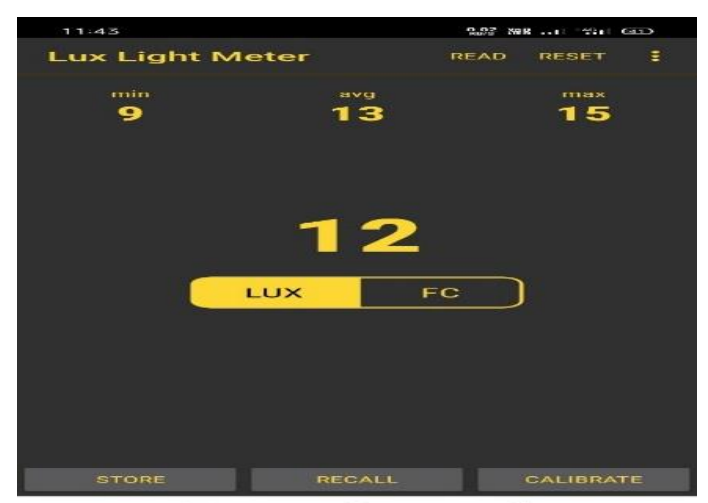

(a)

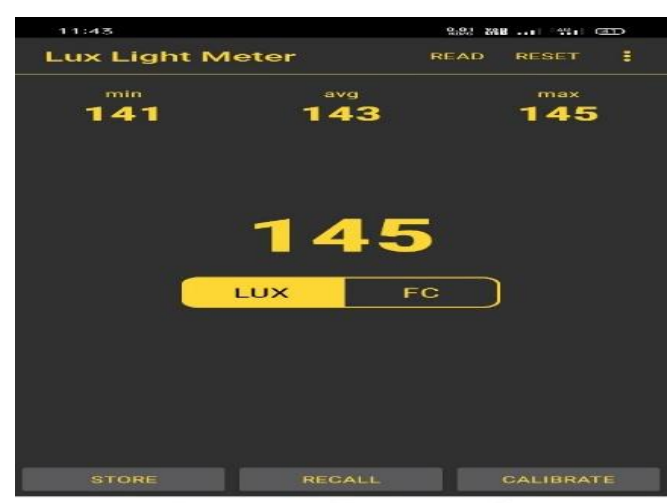

(b) 


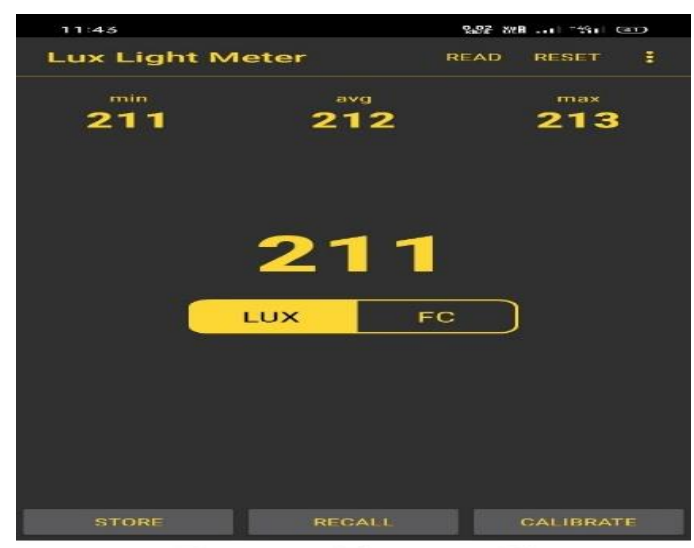

(c)

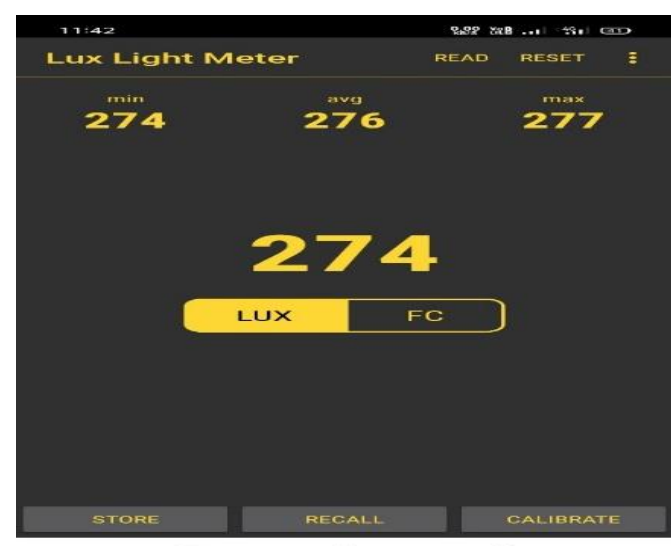

(d)

Fig. 11 (a) Ambient light intensity, (b) Light intensity at dimming value $=0.25$, (c) Light intensity at dimming value $=0.5$ (d) Light intensity at dimming value $=0.75$

TABLE II

Light INTENSITY FoR VARIOUS DimMing VALUE

\begin{tabular}{|c|c|c|c|c|}
\hline \multirow{2}{*}{ Dim Value, $\alpha$} & \multicolumn{3}{|c|}{ Light intensity (Lux) } & \multirow{2}{*}{ Measured at a distance of } \\
\cline { 2 - 4 } & Min. & Avg. & Max. & \\
\hline Ambient condition & 9 & 13 & 15 & - \\
\hline 0.25 & 141 & 143 & 145 & $200 \mathrm{~cm}(2 \mathrm{~m})$ \\
\hline 0.5 & 211 & 212 & 213 & $200 \mathrm{~cm}(2 \mathrm{~m})$ \\
\hline 0.75 & 274 & 276 & 277 & $200 \mathrm{~cm}(2 \mathrm{~m})$ \\
\hline
\end{tabular}

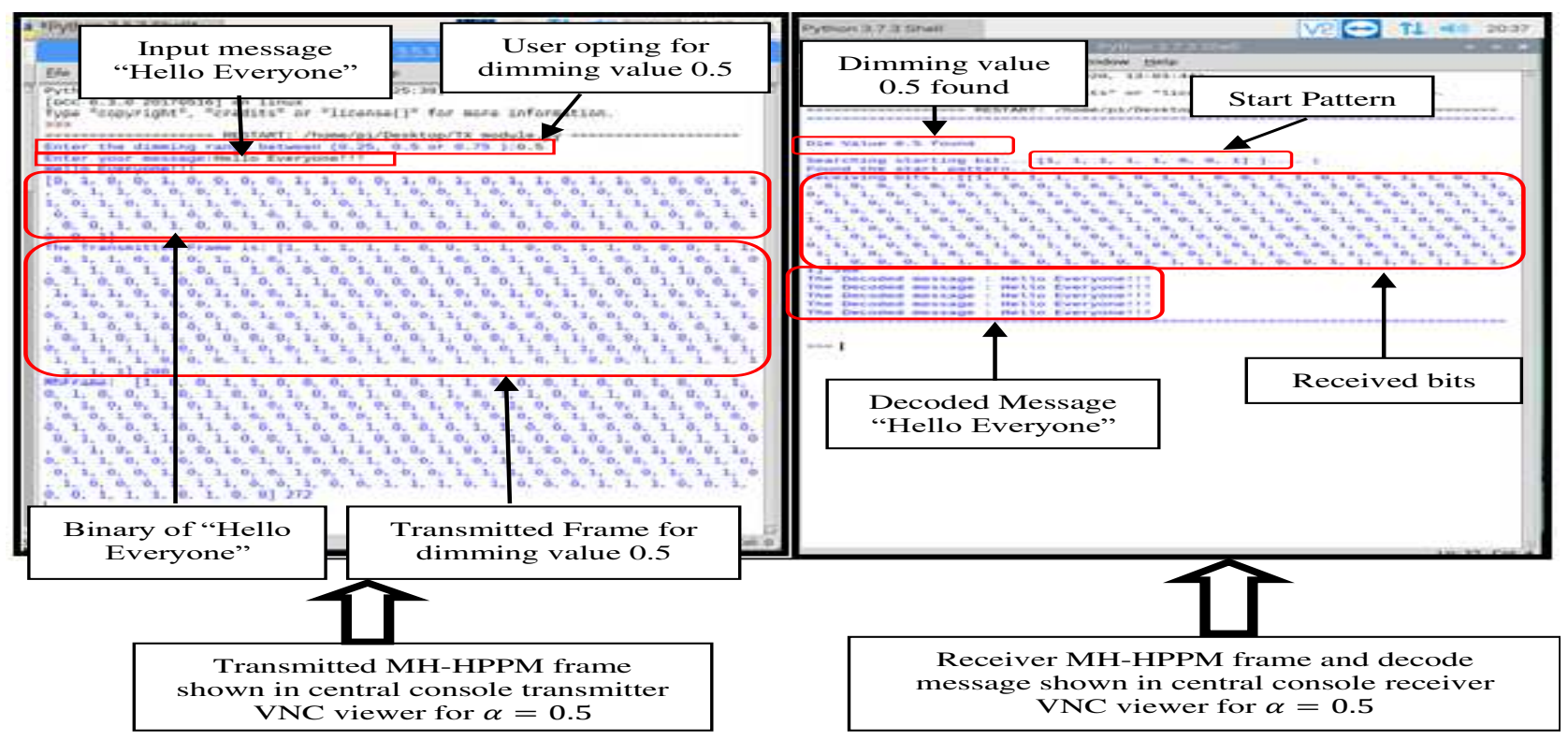

Fig. 12 Showing transmitted MH-HPPM frame $(\alpha=0.5)$, MH-HPPM received frame $(\alpha=0.5)$ and decoded text "Hello Everyone" running in transmitter and receiver $\mathrm{VNC}$ viewer respectively 


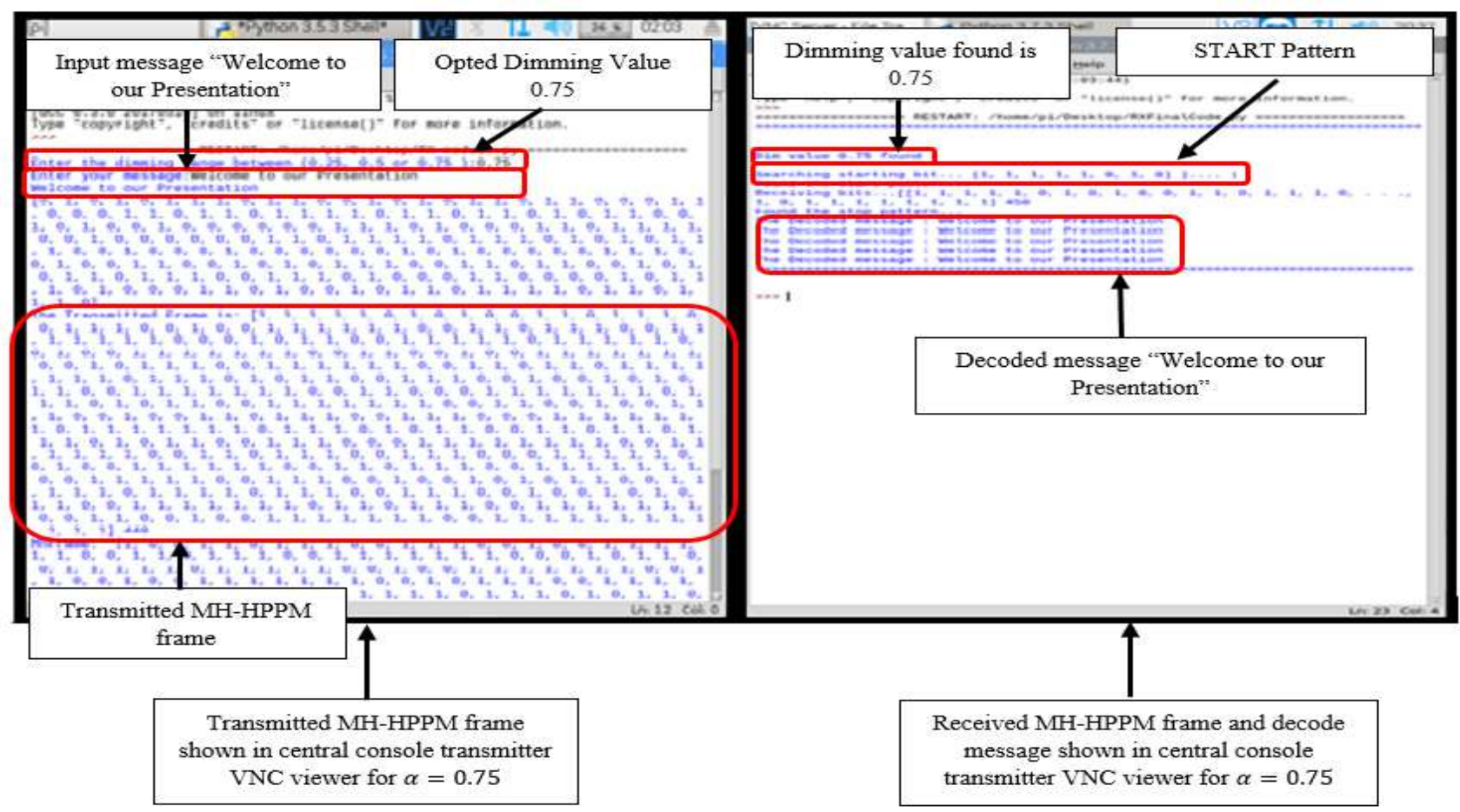

Fig. 13 Showing transmitted MH-HPPM frame $(\alpha=0.775)$, MH-HPPM received frame $(\alpha=0.75)$ and decoded text "Welcome to our Presentation" running in transmitter and receiver VNC viewer respectively

Throughput versus distance: The VLC prototype's throughput is also evaluated by varying the distance between the transmitter and receiver. In the experiments, three dimming levels $(0.25,0.5$, and 0.75) were tested. Each marker in the result plot shown in fig. 14 represents a 30-second experiment. It is observed that the VLC protype maintains its peak throughput at dimming levels 0.5 and 0.75 at distances of up to $3.6 \mathrm{~m}$, and at dimming level 0.25 at distances of up to $3 \mathrm{~m}$. After this distance, the throughput for the respective dimming levels drops dramatically because the received signal strengths are insufficient for the receiver to decode the signal. Given that the height of the ceiling in a typical office/home is usually around 2.5 to $3 \mathrm{~m}$, the communication distance of $3.6 \mathrm{~m}$ supported by VLC prototype for dimming values $0.5,0.75$, and $3 \mathrm{~m}$ for dimming value 0.25 is sufficient in practice. Furthermore, it is also observed that the LED dimming level has no effect on the VLC system's communication distance.

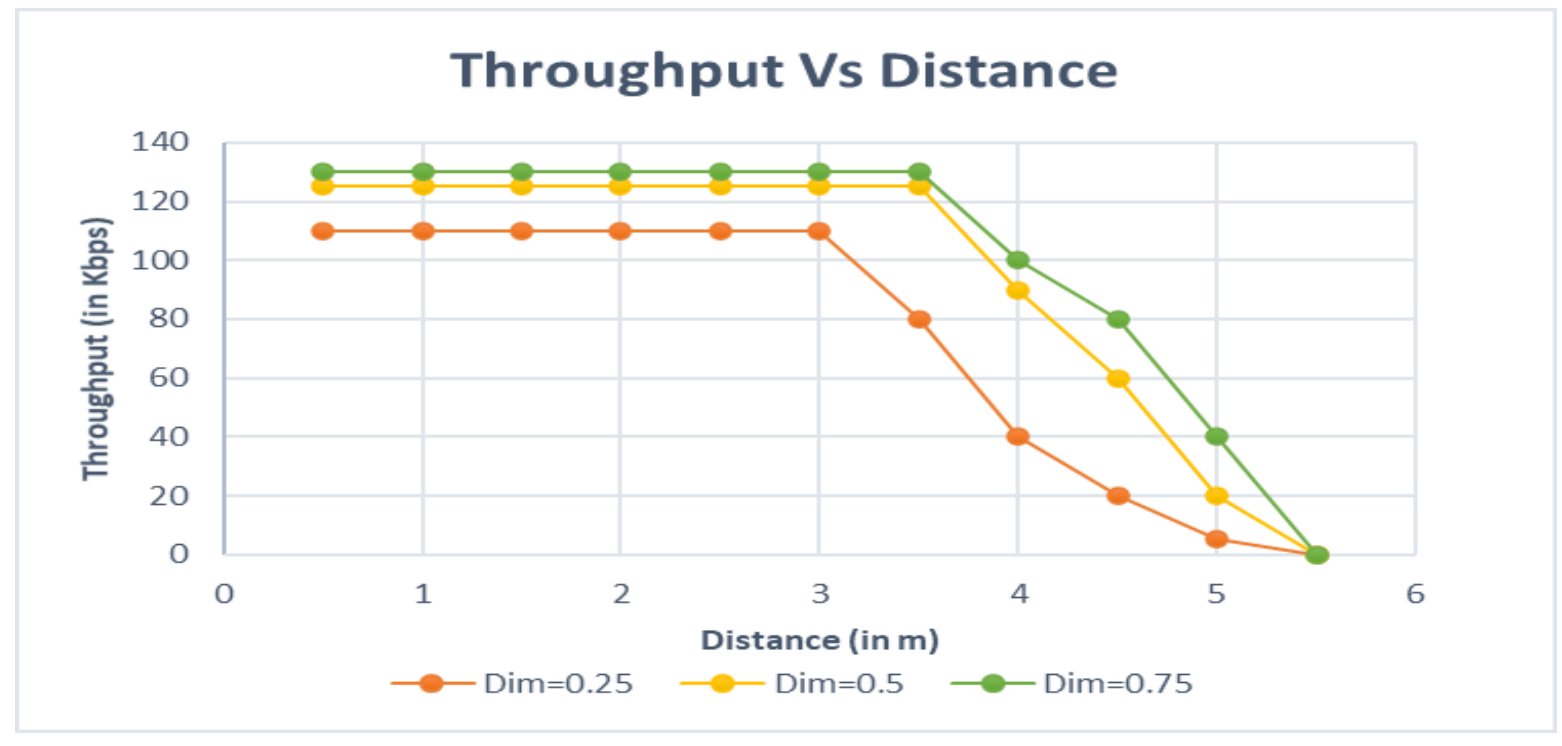

Fig. 14 Throughput Vs Communication distance for various dimming values 


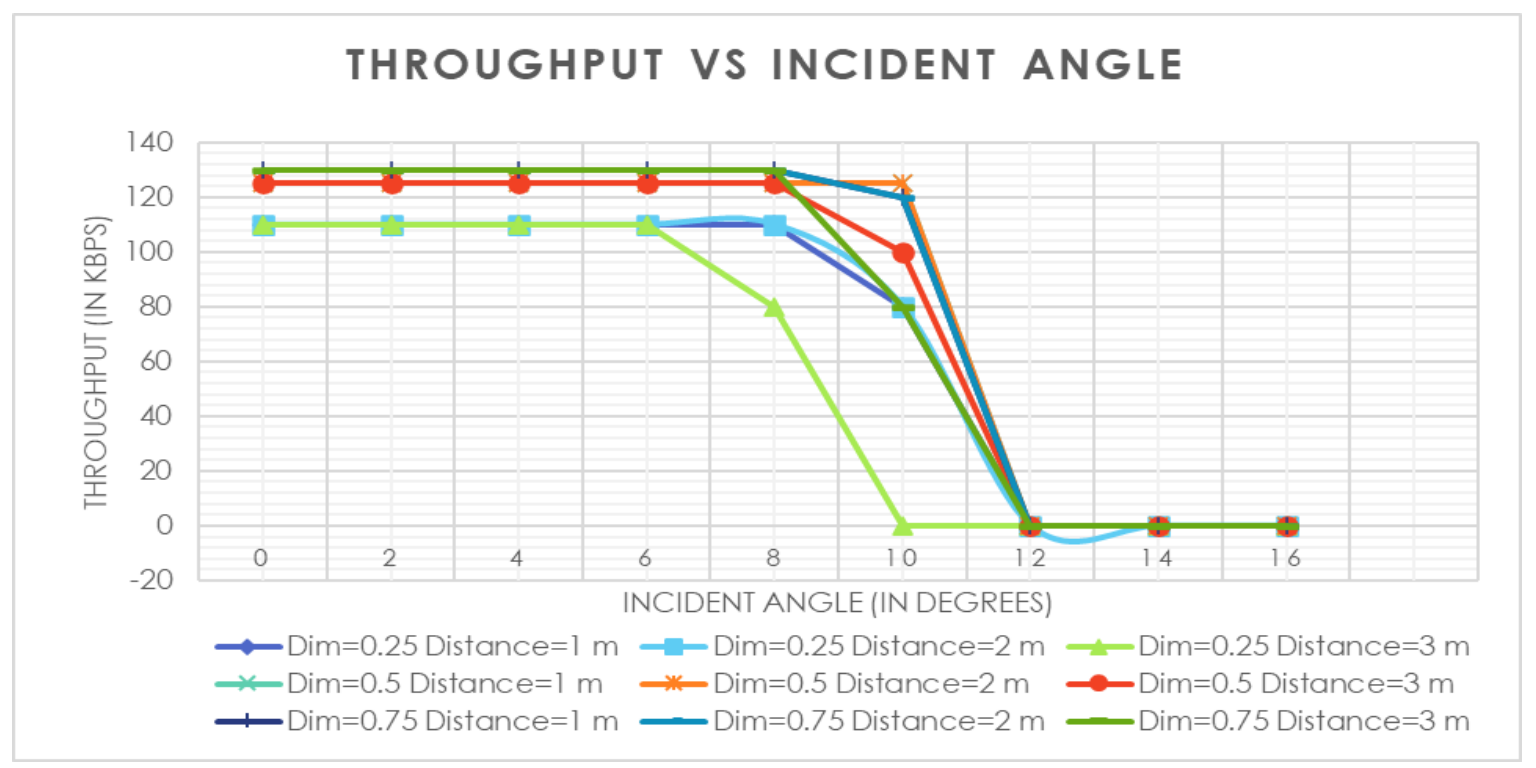

Fig. 15 Throughput Vs Incident angle (degrees) for various dimming values

Throughput versus incident angle: The performance of the presented VLC system is further evaluated under different incident angles. During the experiments, the distance between the transmitter and receiver is kept constant while the incident angles for the various dimming levels are varied. Experiments are carried out at various distances $(1 \mathrm{~m}, 2 \mathrm{~m}$, and $3 \mathrm{~m})$ between the transmitter and receiver. Figure 15 depicts the performance of various dimming levels under different incident angles. The VLC system nearly maintains its performance for different dimming values with LED Field of View (FOV). It is also observed that irrespective of the dimming level longer distance has shorter cut off incident angle. This is due to the fact that the system has already reached the upper bound of the communication distance before the incidence angle begins to affect it.

\section{Conclusions}

Dimming and visible light communication co-design is an important topic. Dimming controlled MH-HPPM is implemented in this paper to achieve different dimming levels without flickering while also achieving and maintaining throughput at each dimming level. The VLC prototype was built with low-cost off-the-shelf components, and extensive testing shows that its performance is adequate for use as an alternative dimming method. As a result, the dimming-controlled MH-HPPM VLC prototype has the potential to pique the interest of additional system-level research efforts on codesigning smart lighting and communication.

\section{Declarations}

Funding- This research is not funded

Conflicts of interest/Competing interests- To our best knowledge this work doesn't have any conflicts of interest

Availability of data and material- No external data is used

Code availability- Code is not available elsewhere except the authors

\section{References}

[1]. T. Komine and M. Nakagawa, "Fundamental analysis for visible light communication system using LED lights," in IEEE Transactions on Consumer Electronics, vol. 50, no. 1, pp. 100-107, Feb. 2004. doi: 10.1109/TCE.2004.1277847

[2]. S. Rajagopal, R. D. Roberts, and S.-K. Lim, "IEEE 802.15.7 visible light communication: modulation schemes and dimming support," IEEE Communications Magazine, vol. 50, no. 3, pp. 72-82, 2012.

[3]. S. M. Berman, D. S. Greenhouse, I. L. Bailey, R. Clear, and T. W. Raasch, "Human electroretinogram responses to 
video displays, fluorescent lighting and other high frequency sources,” Optometry and Vision Science, vol. 68, pp. 645662,1991

[4]. F. Zafar, D. Karunatilaka, R. Parthiban, "Dimming schemes for visible light communication: The state of research", IEEE Wirel. Commun. 22 (2) (2015) 29-35.

[5]. H.L. Sang, S.Y. Jung, J.K. Kwon, "Modulation and coding for dimmable visible light communication," IEEE Commun. Mag. 53 (2) (2015) 136-143.

[6]. Y. Zuo, Jian Zhang, Yan-Yu Zhang, "A spectral-efficient dimming control scheme with multi-level incremental constant weight codes in visible light communication systems," Optics Communications, Vol. 426, 1 November 2018 , pp. 531-534.

[7]. Z. Babar et al., "Unary-Coded Dimming Control Improves ON-OFF Keying Visible Light Communication," in IEEE Transactions on Communications, vol. 66, no. 1, pp. 255-264, Jan. 2018.

[8]. Y. Zuo, J. Zhang, Y. Zhang and R. Chen, "Weight Threshold Check Coding for Dimmable Indoor Visible Light Communication Systems," in IEEE Photonics Journal, vol. 10, no. 3, pp. 1-11, June 2018, Art no. 7904811

[9]. Y. Zuo and J. Zhang, "Dimming control scheme for VLC systems based on multilevel data transmission," Appl. Opt. 57, 9584-9588 (2018).

[10]. Keyan Deng, Yi Wan, Ying Lu, "MPPM based dimming control scheme in visible light communication systems", Optics Communications, 451 (2019), pp. 168-173.

[11]. J. K. Kwon, "Inverse Source Coding for Dimming in Visible Light Communications Using NRZ-OOK on Reliable Links," in IEEE Photonics Technology Letters, vol. 22, no. 19, pp. 1455-1457, Oct.1, 2010.

[12]. Jinyoung Noh, Seungwoo Lee, Jonghun Kim, MinChul Ju, Youngil Park, "A dimming controllable VPPM-based VLC system and its implementation”, Optics Communications, Volume 343, 15 May 2015, Pages 34-37.

[13]. Das, S., Mandal, S.K. Dimming controlled multi header hybrid PPM (MH-HPPM) for visible light communication. Opt Quant Electron 53, 123 (2021). https://doi.org/10.1007/s11082-021-02758-6

[14]. Sergio Sandoval-Reyes, Transmission and Reception of Images via Visible Light, Research in Computing Science 147(12), 2018, pp. 193-202

[15]. Motoi Kodama, Shinichiro Haruyama, "A Fine-Grained Visible Light Communication Position Detection System Embedded in One-Colored Light Using DMD Projector", Mobile Information Systems, vol. 2017, Article ID 9708154,10 pages, 2017. https://doi.org/10.1155/2017/9708154

[16]. Khalid, A., Saeed, A., Altaf, Z., Siddiqui, A. R., Khan, N., Khan, N., \& Ali, A. (2019). Design of a CSK-CDMA Based Indoor Visible Light Communication Transceiver using Raspberry Pi and LabVIEW. International Journal of Integrated Engineering, 11(8), 119-125

[17]. T. Wang and Z. Zhao, "An implementation of visible light communication based on Raspberry Pi," 2018 IEEE Integrated STEM Education Conference (ISEC), 2018, pp. 218-219, doi: 10.1109/ISECon.2018.8340486.

[18]. Scheianu, A., Suciu, G., Drosu, A., Petre, I., \& Miu, D. (2020). Hybrid VLC Communications System for Increased Security Based on Raspberry $\mathrm{Pi}$ Microcomputer. Annals of Disaster Risk Sciences, 3(1). https://doi.org/10.51381/adrs.v3i1.44 